\title{
A SURVEY OF SPIDERS FOUND IN FALLEN PINE CONES IN EASTERN WASHINGTON STATE
}

\author{
Laurel J. Ramseyer ${ }^{1}$ and Rodney L. Crawford ${ }^{2}$
}

\begin{abstract}
Aвstract.-Fallen pine cones are a microhabitat for spiders in Washington State. Eighty-nine species of spiders from 24 families and 70 genera were identified from 1060 specimens collected from over 4567 cones of Pinus ponderosa and Pinus monticola between 2008 and 2013. Euryopis formosa Banks 1908 (Theridiidae) and Pholcophora americana Banks 1896 (Pholcidae) were the most abundant species collected (27\% and $9.3 \%$ of identified specimens, respectively) and occurred most frequently (49\% and $21 \%$ of sampling sites, respectively). Fallen cones may be an important microhabitat for these species in Washington. Fallen cones produced 18 spider species rare in Washington, including Theridion rabuni Chamberlin and Ivie 1944 (Theridiidae), which has not been found in any other microhabitat in the state. Sampling fallen cones added a mean of 3 species (SD 2, range 0-9) to site lists created by conventional collecting methods like sweeps, litter sifting, and foliage beats. Agelenid spiders incorporated entire cones into their webs, while other species placed egg sacs or retreats on cone surfaces or used the inner spaces of cones to build prey capture webs or to molt.
\end{abstract}

RESUMEN.-Encontramos que las piñas caídas son un microhábitat para las arañas en el estado de Washington. Se identificaron 89 especies de arañas de 24 familias y 70 géneros en 1,060 muestras obtenidas de más de 4,567 piñas de Pinus ponderosa y Pinus monticola entre los años 2008 y 2013. Las especies colectadas más abundantes fueron Euryopis formosa Banks 1908 (Theridiidae) y Pholcophora americana Banks 1896 (Pholcidae), (27\% y 9.3\% de las muestras identificadas, respectivamente) y encontradas con mayor frecuencia ( $49 \%$ y $21 \%$ de los sitios de muestreo, respectivamente); las piñas caídas podrían representar un microhábitat importante para estas especies en Washington. Las piñas caídas mostraron 18 especies de arañas poco comunes en Washington, incluyendo Theridion rabuni Chamberlin e Ivie 1944 (Theridiidae), que no se ha encontrado en ningún otro microhábitat en el estado. La toma de muestras de piñas caídas añadió un promedio de 3 (DE 2 , entre 0 y 9 ) de especies a las listas de sitios creadas por los métodos convencionales de recolección tales como redadas, tamizado de arena y golpeo de follaje. Las arañas Agelenid incorporaron piñas enteras en sus telarañas, mientras que otras especies colocaron sacos de huevos o crearon lugares de retiro en las superficies o utilizaron los espacios internos de las piñas para construir redes para capturar presas o para mudar de piel.

Different collection methods yield different sets of spider species (Coddington et al. 1996). Making a local species list as complete as possible therefore requires sampling methods complementary enough to access every spider microhabitat. A limitation faced by spider collectors is the ability to recognize "new" spider microhabitats and develop methods to sample them. We discovered that the fallen seed cones of ponderosa pine (Pinus ponderosa Laws.) and western white pine (Pinus monticola Douglas) are a spider microhabitat in Washington State, and we developed a collecting technique to sample the cones. This paper describes the results of a 6-year survey of the spider fauna of fallen cones in Washington. Our goals were to determine which spider species use fallen cones, whether sampling cones was complementary or redundant to other sampling methods, whether any species appeared to focus its activities on cones, and the ways spiders use cones.

\section{Methods}

We collected spiders from the fallen seed cones of P. ponderosa and P. monticola in Washington State from May 2008 through October 2013. We sampled cones at 70 sites located east of the crest of the Cascade Range ("eastern Washington"; Fig. 1), and resampled 2 sites approximately 10 months after the initial sampling date, making 72 sampling events in all. Numbers of sites sampled in the years 2008 through 2013 were 2, 4, 5, 42, 1, and 18 , respectively.

\section{Study Area}

Cones of P. ponderosa and P. monticola were sampled at 64 and 7 sites, respectively.

${ }^{16401}$ Sand Point Way NE, Seattle, WA 98115.

${ }^{2}$ Corresponding author. Burke Museum, University of Washington, Box 353010, Seattle, WA 98195-3010. E-mail: tiso@u.washington.edu 


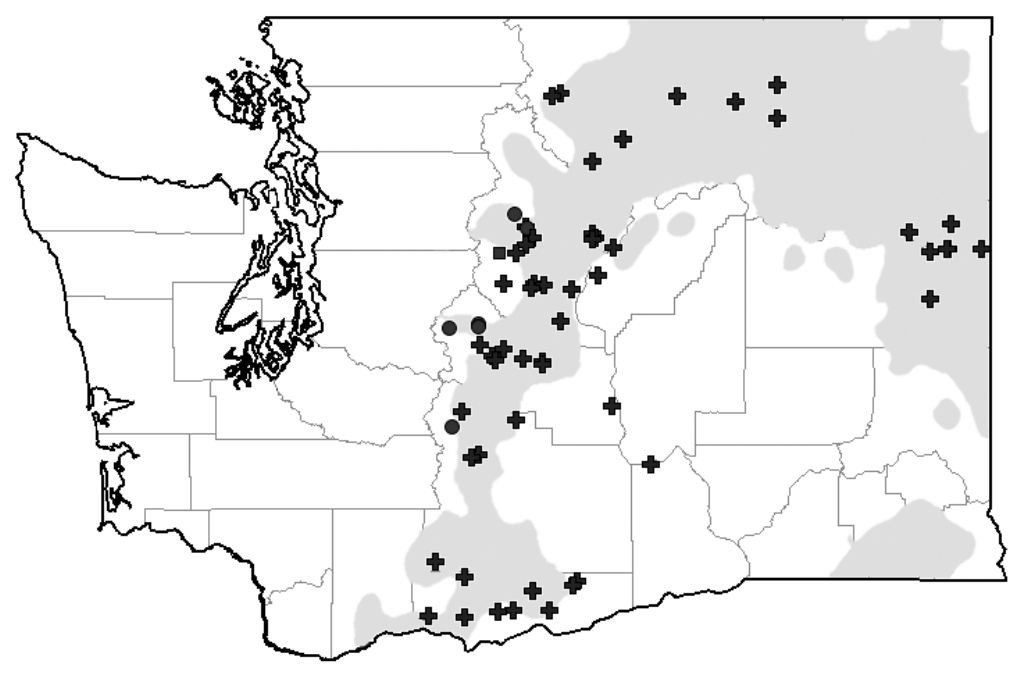

Fig. 1. Map of Washington State showing sites where spiders were collected from the fallen cones of Pinus ponderosa (crosses, 64 sites), P. monticola (circles, 7 sites), or both P. ponderosa and P. monticola (square, 1 site). Shaded area indicates approximate distribution of P. ponderosa in eastern Washington (Kotok 1973).

Pinus ponderosa co-occurred with P. monticola at one site. Most cone sampling sites fell within the "Pinus ponderosa Zone" described by Franklin and Dyrness (1973). The trees at most sites appeared to be naturally occurring, except for the ponderosa pines that were planted as shade trees at 2 sites in a largely treeless section of the Columbia Plateau in central Washington. Site elevations varied from $120 \mathrm{~m}$ to $1460 \mathrm{~m}$.

In eastern Washington, a zone of relatively pure $P$. ponderosa forest typically occurs as one moves upward from treeless steppe into forested mountains (Daubenmire and Daubenmire 1968, Franklin and Dyrness 1973). Most often, the lowest pines are in canyons, whereas occurrence on ridges begins significantly higher. Farther into the mountains, this zone gives way to a Douglas-fir (Pseudotsuga menziesii [Mirb.] Franco) zone, but pine stands occur variably in that zone as well. Many $P$. ponderosa stands have a grass-dominated understory, with Festuca idahoensis Elmer or Agropyron spicatum Pursh dominant in undisturbed stands and species of Poa, invasive Bromus, and others dominant after grazing disturbance. Shrub understory associations feature Symphoricarpos albus (L.) S.F. Blake, Purshia tridentata (Pursh) DC or Ceanothus velutinus Douglas ex Hook. (Daubenmire and Daubenmire 1968). Pinus monticola is not a dominant climax species anywhere in the region, but it is a major seral tree in the midelevation Abies grandis (Douglas ex D. Don) Lindl. and Tsuga heterophylla (Raf.) Sarg. zones of eastern Washington. The species is often associated with $P$. ponderosa and is a minor component of forests throughout the Pacific Northwest (Franklin and Dyrness 1973).

\section{Sampling}

Cones were beaten one at a time into a sweep net (38 cm diameter Heavy Duty Sweep Net bag from BioQuip ${ }^{\circledR}$, made of sailcloth, not mesh). Each cone was held apex-downward as close to its base as possible and given at least 5 solid whacks against the inner wall of the net, which was held flush against the collector's leg to provide a solid striking surface. The cone was rotated once or twice during this procedure. In a preliminary test of the efficiency of this method, we expelled spiders from each of $76 \mathrm{P}$. ponderosa cones as described above, then removed the cone scales with pliers to find any remaining spiders (data not shown). In this test, our beating method produced $95 \%$ of the total number of spiders (84) collected by beating and scale removal combined.

We sampled a minimum of 50 cones per site, cone availability permitting. The number of cones sampled per site was not recorded for the first 8 sites. Cones were randomly selected from the forest floor without regard to their 
state of decay, provided they still retained their scales. Only cones with at least half of their scales open were sampled. Sampled cones were found in various dispositions, including lying on bare soil, lying on needle litter, or partially to fully embedded in litter. We sampled cones between March and November, with most sampling events occurring in June (14), July (17), and October (28). Cone sampling times varied between midmorning and dusk.

To understand how spiders use fallen cones, many cones sampled in 2011 and 2013 were visually examined for the presence of silk structures or spiders before being beaten into the sweep net. We attempted to photograph any spiders noted in situ or after being expelled from the cone. These observations were sporadic and were not quantified.

\section{Specimen Identification}

Relevant literature and R.L. Crawford's file of genitalia drawings were used to identify the specimens. Juveniles were identified to species when possible, with varying degrees of reliability. Family and some genus nomenclature follows Crawford (1988), and species nomenclature follows World Spider Catalog (2014). Voucher specimens are deposited in the arachnology collection at the Burke Museum of Natural History and Culture located at the University of Washington in Seattle, Washington.

\section{Analysis}

Results of the present survey were compared to the Burke Museum's Washington spider collection ("Burke Collection"), which contains over 109,000 specimens representing 39 families, 225 genera, and approximately 920 species collected from 1890 to present.

The number of specimens per cone was calculated by dividing the number of specimens collected by the number of cones sampled during a sampling event. A $t$ test was used to test the null hypothesis that mean specimens per cone for $P$. ponderosa and $P$. monticola samples were not different.

We defined taxon abundance as the number of individuals identified to family or species collected during the survey period. Occurrence was calculated by dividing the number of sites where a family or species was found by the total number of sites sampled $(n=70)$. To determine whether family abundance rankings were different for samples collected from the fallen cone and non-cone microhabitats, we calculated the percentage of specimens identified to species from each family for spiders collected from fallen cones at all sites $(n=70)$ and for spiders in the Burke Collection collected during the same period from groundlevel non-cone microhabitats at cone sites $(n=39)$. The latter group included spiders active on the ground, under or between rocks, in or under objects on the ground, in ground surface webs, sifted from litter, or sifted from moss on rocks. We estimated spider species richness for the fallen cone microhabitat by calculating the Chao 1 estimator using EstimateS (Colwell 2013). We did not include exuviae in any calculations.

Abundance and occurrence calculations were made using the sum total of adult specimens and juvenile specimens identified to species. Juvenile Pholcophora americana and Euryopsis formosa are more readily identifiable to species than juveniles of many other Washington spiders because other species of these genera are seldom, if ever, present (R.L. Crawford personal observation). To test whether inclusion of identified juveniles influenced species rankings, we recalculated species abundance and occurrence values using only adult specimen counts.

Dice's coefficient of association (Dice 1945) was used to test whether $E$. formosa and $P$. americana co-occurred at the same sites more frequently than would be expected by random chance.

To determine how the fallen cone microhabitat added to site species lists, we counted the number of species collected from fallen cones that were not collected from other microhabitats at a sample site. The non-cone microhabitats sampled depended on each site's characteristics, but typically included leaf litter, conifer tree foliage, sweepable grass and herbs, shrub foliage, bark, exposed surfaces, the undersides of stones and other objects on the ground, and dead wood. We did not quantify sampling effort for non-cone microhabitat collections, but such effort was roughly similar at most sites.

We recorded qualitative observations on and photographed the disposition of cones and associated litter at most sampling sites.

Unless otherwise stated, calculations were made using Microsoft Excel 2000 descriptive statistics functions. 


\section{RESUlts}

In all, we collected 1060 spiders representing 24 families, 70 genera, and 89 species (Table 1). One thousand fifty-five specimens were identified to family, 950 to genus, and 542 to species. Of the spiders collected, $17 \%$ were mature females, $7 \%$ were mature males, and $76 \%$ were juveniles. Forty-four percent of species identified were represented by singletons. The Chao 1 estimate of species richness for the fallen cone microhabitat at the sampling sites was 143.2 species (SD 23.8, 95\% CI 112.8-212.4).

We sampled more than 4567 fallen pine cones, of which 4256 (93\%) were from $P$. ponderosa and 311 (7\%) from P. monticola. The number of cones sampled per site varied from 20 to $240(\bar{x}=71$, SD 39, $n=64)$. Cone availability limited the number of cones sampled to fewer than 50 cones per site at 8 sites.

The mean number of spiders collected per cone was not significantly different for samples taken at $P$. ponderosa sites $(n=59)$ and $P$. monticola sites $(n=5)(t=0.9195, \mathrm{df}=62$, $P>0.05)$. Consequently, we pooled the data on spiders per cone for both species of pine. Based on the combined data from 64 sites, we collected a mean of 0.24 (SD 0.26) specimens per cone. Number of spiders per cone varied widely between 0.020 and 1.3 spiders (median $0.15)$ at different sites.

Abundance and occurrence of specimens identified to family are given in Table 2 . The most abundant families collected from fallen cones were Theridiidae (23\%), Gnaphosidae (13\%), and Linyphiidae (13\%). The most frequently occurring family was Theridiidae, with specimens collected at $67 \%$ of sampling sites, followed by Linyphiidae (57\%) and Gnaphosidae $(54 \%)$. The most abundant spider families collected from ground-level non-cone microhabitats at cone sampling sites were Linyphiidae $(44 \%)$, Lycosidae $(9.1 \%)$, and Corinnidae (8.3\%) (Table 2).

Euryopis formosa Banks 1908 was the most abundant species found in fallen cones, with 145 specimens (27\% of total identified to species) collected, followed by Pholcophora americana Banks 1896 (50, 9.3\%) and Meioneta fillmorana (Chamberlin 1919) (27, 5.0\%) (Table 1). The 63 Dictyna calcarata Banks 1904 specimens $(12 \%)$ were all from one collection site, so we considered the statistic an outlier and removed it from the ranking. Euryopis formosa was also the most frequently occurring species, found at $47 \%$ of sampling sites, followed by P. americana (21\%) and M. fillmorana (13\%) (Table 1).

Excluding juveniles from the species abundance calculation did not alter $E$. formosa's ranking as the most abundant species (46 adult specimens, 18\%), but it did elevate M. fillmorana (whose juveniles, if present, could not be assigned to species) from third to second place $(27,11 \%)$ and dropped $P$. americana from second to third place $(22,8.7 \%)$. Excluding juveniles from the occurrence calculations did not affect the occurrence rankings.

Euryopis formosa and $P$. americana were both found in fallen cones at 12 of the same sampling sites. The 2 species co-occurred $60 \%$ more frequently than expected by chance $\left(\chi^{2}=6.821, \mathrm{df}=1, P<0.01\right.$, coefficient of association $=1.6$ ).

We collected 2 species from fallen cones not recorded previously in Washington: Theridion rabuni Chamberlin and Ivie 1944 and Meioneta cf. delphina (Dupérré 2013), the latter subsequently found in pine needle litter. Theridion rabuni is presently the only spider species found exclusively in fallen cones in Washington. In addition, fallen cones yielded specimens of 16 other state rarities, 7 of which are as yet undescribed (Table 1).

At sites with a full range of microhabitats sampled, the fallen cone microhabitat added a mean of 3 species ( $\mathrm{SD} 2$, median $=3$, range $0-9, n=48$ sites) to the full site species list. This addition represents a mean of $20 \%$ (SD $16 \%$, median $=16 \%$, range $0 \%-75 \%, n=48$ sites) of the total number of species collected at a site.

The present survey more than quadrupled the number of $E$. formosa specimens in the Burke Collection and doubled the number of sites in Washington from which the species has been collected. Similarly, collecting from fallen cones increased both specimens and collection sites of $P$. americana in the Burke Collection by one third.

The current survey, along with records in the Burke Collection, indicate that in Washington $E$. formosa primarily occurs east of the Cascade Range crest, largely in association with pines (Fig. 2). All specimens from fallen cones and all but one specimen in the Burke Collection from non-cone microhabitats were 
TABLE 1. List of spider species collected from fallen pine cones in eastern Washington State. Species status is relative to the Burke Collection. Status abbreviations: $\mathrm{U}=$ uncommon (found in 10 or fewer locations in Washington), $\mathrm{R}=\mathrm{rare}$ ( 5 or fewer), $\mathrm{ER}=$ exceedingly rare $(1$ or 2$), \mathrm{O}=$ species found only in fallen cones in Washington.

\begin{tabular}{|c|c|c|c|}
\hline Family/species & $\begin{array}{c}\text { Status in } \\
\text { Washington }\end{array}$ & $\begin{array}{l}\text { Number of sites } \\
\text { collected from } \\
\text { (Pinus ponderosa- } \\
\text { P. monticola) }\end{array}$ & $\begin{array}{c}\text { Number of } \\
\text { specimens } \\
\text { (females-males- } \\
\text { juveniles) }\end{array}$ \\
\hline \multicolumn{4}{|l|}{ AGELENIDAE (SENSU LATO) } \\
\hline Agelenopsis sp. & & $1(1-0)$ & $1(0-0-1)$ \\
\hline Agelenopsis utahana (Chamberlin and Ivie 1933) & & $1(1-0)$ & $1(1-0-0)$ \\
\hline Calymmaria nana (Simon 1897) & & $1(1-0)$ & $3(2-0-1)$ \\
\hline Calymmaria sp. & & $1(1-0)$ & $2(0-0-2)$ \\
\hline Cryphoeca exlineae Roth 1988 & & $4(2-2)$ & $9(5-0-4)$ \\
\hline Hololena nedra Chamberlin and Ivie 1942 & & $1(1-0)$ & $1(1-0-0)$ \\
\hline Novalena intermedia (Chamberlin and Gertsch 1930) & & $1(1-0)$ & $6(0-0-6)$ \\
\hline Novalena sp. & & $6(4-2)$ & $12(0-0-12)$ \\
\hline Tegenaria agrestis (Walckenaer 1802) & & $1(1-0)$ & $1(0-0-1)$ \\
\hline Tegenaria sp. & & $1(1-0)$ & $1(0-0-1)$ \\
\hline Unidentified juveniles & & $5(5-2)$ & $6(0-0-6)$ \\
\hline \multicolumn{4}{|l|}{ AMAURoBIIDAE } \\
\hline Callobius canada (Chamberlin and Ivie 1947) & & $2(2-0)$ & $2(2-0-0)$ \\
\hline Callobius nevadensis (Simon 1884) & & $1(1-0)$ & $2(1-0-1)$ \\
\hline Callobius sp. & & $7(5-2)$ & $17(0-0-17)$ \\
\hline Zanomys aquilonia Leech 1972 & & $2(1-1)$ & $8(6-0-2)$ \\
\hline Zanomys kaiba Chamberlin 1948 & & $1(1-0)$ & $2(2-0-0)$ \\
\hline \multicolumn{4}{|l|}{ ANYPHAENIDAE } \\
\hline Anyphaena pacifica (Banks 1896) & & $1(1-0)$ & $1(1-0-0)$ \\
\hline Anyphaena sp. & & $13(13-0)$ & $36(0-0-36)$ \\
\hline \multicolumn{4}{|l|}{ ARANEIDAE } \\
\hline Cyclosa conica (Pallas 1772) & & $1(1-0)$ & $1(0-0-1)$ \\
\hline Metepeira sp. & & $1(1-0)$ & $1(0-0-1)$ \\
\hline \multicolumn{4}{|l|}{ Clubionidae } \\
\hline Clubiona mutata Gertsch 1941 & $\mathrm{R}$ & $1(1-0)$ & $1(0-1-0)$ \\
\hline Clubiona sp. & & $2(2-0)$ & $2(0-0-2)$ \\
\hline \multicolumn{4}{|l|}{ CORINNIDAE } \\
\hline Castianeira longipalpa (Hentz 1847) & & $2(2-0)$ & $4(1-0-3)$ \\
\hline Phrurotimpus borealis (Emerton 1911) & & $3(3-0)$ & $20(5-1-14)$ \\
\hline Phrurotimpus certus Gertsch 1941 & & $2(2-0)$ & $9(2-3-4)$ \\
\hline Phrurotimpus parallelus (Chamberlin 1921) & & $2(2-0)$ & $4(1-1-2)$ \\
\hline Phrurotimpus sp. & & $5(5-0)$ & $14(0-0-14)$ \\
\hline \multicolumn{4}{|l|}{ CYBAEIDAE } \\
\hline Cybaeota nana Chamberlin and Ivie 1937 & & $1(0-1)$ & $1(1-0-0)$ \\
\hline Cybaeus sp. & & $1(1-0)$ & $2(0-0-2)$ \\
\hline \multicolumn{4}{|l|}{ DICTYNIDAE } \\
\hline Dictyna calcarata Banks 1904 & & $1(1-0)$ & $63(8-0-55)$ \\
\hline Dictyna chitina Chamberlin and Gertsch 1958 & & $1(1-0)$ & $1(1-0-0)$ \\
\hline Dictyna reticulata Gertsch and Ivie 1936 & $\mathrm{U}$ & $1(1-0)$ & $4(0-1-3)$ \\
\hline Dictyna sp. \#4 & & $1(1-0)$ & $1(1-0-0)$ \\
\hline Dictyna sp. \#9 & $\mathrm{R}$ & $3(3-0)$ & $5(4-0-1)$ \\
\hline Dictyna sp. \#13 & ER & $1(1-0)$ & $1(0-1-0)$ \\
\hline Dictyna uintana Chamberlin 1919 & & $1(1-0)$ & $2(1-0-1)$ \\
\hline Dictyna sp. & & $9(9-0)$ & $15(0-0-15)$ \\
\hline Mallos pallidus (Banks 1904) & $\mathrm{U}$ & $1(1-0)$ & $2(2-0-0)$ \\
\hline Unidentified juveniles & & $3(3-0)$ & $11(0-0-11)$ \\
\hline \multicolumn{4}{|l|}{ GNAPHOSIDAE } \\
\hline Callilepis pluto Banks 1896 & & $2(1-1)$ & $2(2-0-0)$ \\
\hline Callilepis sp. & & $2(1-1)$ & $3(0-0-3)$ \\
\hline Drassodes sp. & & $4(4-0)$ & $7(0-0-7)$ \\
\hline Drassyllus sp. & & $2(2-0)$ & $3(0-0-3)$ \\
\hline Gnaphosa sp. & & $16(16-0)$ & $73(0-0-73)$ \\
\hline Haplodrassus eunis Chamberlin 1922 & $\mathrm{U}$ & $1(1-0)$ & $1(0-1-0)$ \\
\hline Haplodrassus sp. & & $1(1-0)$ & $1(0-0-1)$ \\
\hline Micaria sp. & & $9(8-1)$ & $16(0-0-16)$ \\
\hline Poecilochroa columbiana Emerton 1917 & & $7(7-0)$ & $11(1-0-10)$ \\
\hline Poecilochroa montana Emerton 1890 & & $5(5-0)$ & $6(2-0-4)$ \\
\hline
\end{tabular}


TABLE 1. Continued.

\begin{tabular}{|c|c|c|c|}
\hline Family/species & $\begin{array}{c}\text { Status in } \\
\text { Washington }\end{array}$ & $\begin{array}{l}\text { Number of sites } \\
\text { collected from } \\
\text { (Pinus ponderosa- } \\
\text { P. monticola) }\end{array}$ & $\begin{array}{c}\text { Number of } \\
\text { specimens } \\
\text { (females-males- } \\
\text { juveniles) }\end{array}$ \\
\hline Zelotes sp. & & $9(9-0)$ & $12(0-0-12)$ \\
\hline Unidentified juveniles & & $6(6-0)$ & $10(0-0-10)$ \\
\hline \multicolumn{4}{|l|}{ HAHNIIDAE } \\
\hline Hahnia sanjuanensis Exline 1938 & ER & $1(1-0)$ & $3(1-0-2)$ \\
\hline \multicolumn{4}{|l|}{ LINYPHIIDAE, ERIGONINAE } \\
\hline Disembolus procerus Millidge 1981 & ER & $1(1-0)$ & $1(0-1-0)$ \\
\hline Disembolus sp. \#2 & $\mathrm{R}$ & $2(2-0)$ & $5(4-1-0)$ \\
\hline Disembolus sp. \#11 & ER & $1(1-0)$ & $1(1-0-0)$ \\
\hline Erigone dentosa O. P.-Cambridge 1894 & & $1(1-0)$ & $1(1-0-0)$ \\
\hline Pocadicnemis occidentalis Millidge 1976 & & $3(2-1)$ & $3(3-0-0)$ \\
\hline Spirembolus demonologicus (Crosby, in Chamberlin 1925) & & $1(1-0)$ & $1(1-0-0)$ \\
\hline Spirembolus mundus Chamberlin and Ivie 1933 & & $5(5-0)$ & $7(4-3-0)$ \\
\hline Tachygyna exilis Millidge 1984 & $\mathrm{U}$ & $1(0-1)$ & $2(2-0-0)$ \\
\hline \multicolumn{4}{|l|}{ LINYPHIIDAE, LiNYPHIINAE (INCLUDING MiCRONETINAE) } \\
\hline Agyneta protrudens (Chamberlin and Ivie 1933) & & $1(0-1)$ & $1(1-0-0)$ \\
\hline Lepthyphantes pollicaris Zorsch 1937 & & $1(1-0)$ & $1(1-0-0)$ \\
\hline Lepthyphantes sp. & & $1(0-1)$ & $1(0-0-1)$ \\
\hline Linyphantes pualla Chamberlin and Ivie 1942 & & $1(0-1)$ & $1(1-0-0)$ \\
\hline Meioneta bucklei (Dupérré 2013) & & $4(4-0)$ & $6(3-3-0)$ \\
\hline Meioneta crawfordi (Dupérré 2013) & ER & $1(1-0)$ & $1(0-1-0)$ \\
\hline Meioneta danielbelangeri (Dupérré 2013) & $\mathrm{R}$ & $1(1-0)$ & $1(1-0-0)$ \\
\hline Meioneta cf. delphina (Dupérré 2013) & $\mathrm{R}$ & $2(2-0)$ & $3(1-2-0)$ \\
\hline Meioneta fillmorana (Chamberlin 1919) & & $9(9-0)$ & $27(27-0-0)$ \\
\hline Meioneta fratrella (Chamberlin 1919) & $\mathrm{U}$ & $1(1-0)$ & $2(0-2-0)$ \\
\hline Meioneta ordinaria Chamberlin and Ivie 1947 & & $3(1-2)$ & $5(5-0-0)$ \\
\hline Meioneta simplex (Emerton 1926) & & $5(4-1)$ & $5(2-2-1)$ \\
\hline Meioneta vinki (Dupérré 2013) & $\mathrm{U}$ & $2(2-0)$ & $3(2-1-0)$ \\
\hline Neriene litigiosa (Keyserling 1886) & & $1(1-0)$ & $1(0-0-1)$ \\
\hline Tennesseellum formicum (Emerton 1882) & & $1(1-0)$ & $1(0-0-1)$ \\
\hline Unidentified linyphiid juveniles & & $20(16-4)$ & $62(0-0-62)$ \\
\hline \multicolumn{4}{|l|}{ LIOCRANIDAE } \\
\hline Agroeca ornata Banks 1892 & & $2(1-1)$ & $2(2-0-0)$ \\
\hline Unidentified juveniles & & $1(1-0)$ & $1(0-0-1)$ \\
\hline \multicolumn{4}{|l|}{ LYCOSIDAE } \\
\hline Pardosa mackenziana (Keyserling 1877) & & $1(1-0)$ & $1(1-0-0)$ \\
\hline Pardosa sp. & & $14(12-2)$ & $26(0-0-26)$ \\
\hline Tarentula kochii Keyserling 1876 & & $2(2-0)$ & $3(2-0-1)$ \\
\hline Tarentula sp. & & $5(4-1)$ & $5(0-0-5)$ \\
\hline \multicolumn{4}{|l|}{ MiMETIDAE } \\
\hline Mimetus hesperus Chamberlin, 1923 & & $1(1-0)$ & $1(0-0-1)$ \\
\hline \multicolumn{4}{|l|}{ MYSMENIDAE } \\
\hline Trogloneta sp. \#2 & & $1(1-0)$ & $1(0-1-0)$ \\
\hline Trogloneta sp. & & $2(2-0)$ & $3(0-0-3)$ \\
\hline \multicolumn{4}{|l|}{ OONOPIDAE } \\
\hline Orchestina sp. \#1 & $\mathrm{R}$ & $1(1-0)$ & $1(0-1-0)$ \\
\hline \multicolumn{4}{|l|}{ PHOLCIDAE } \\
\hline Pholcophora americana Banks 1896 & & $15(15-0)$ & $50(13-9-28)$ \\
\hline \multicolumn{4}{|l|}{ PimoIDAE } \\
\hline Pimoa sp. & & $5(2-3)$ & $7(0-0-7)$ \\
\hline \multicolumn{4}{|l|}{ SALTICIDAE } \\
\hline Evarcha proszynskii Marusik and Logunov 1998 & & $3(3-0)$ & $4(1-2-1)$ \\
\hline Habronattus americanus (Keyserling 1885) & & $1(1-0)$ & $1(1-0-0)$ \\
\hline Habronattus sansoni (Emerton 1915) & $\mathrm{U}$ & $1(1-0)$ & $2(1-1-0)$ \\
\hline Habronattus sp. \#3 & ER & $1(1-0)$ & $1(0-1-0)$ \\
\hline Neon pixii Gertsch and Ivie 1955 & ER & $1(1-0)$ & $5(1-0-4)$ \\
\hline Neon sp. \#1 (near N. pixii) & $\mathrm{U}$ & $4(4-0)$ & $5(4-1-0)$ \\
\hline Neon sp. & & $5(5-0)$ & $9(0-0-9)$ \\
\hline Pelegrina sp. & & $1(1-0)$ & $1(0-0-1)$ \\
\hline Phanias watonus (Chamberlin and Ivie, 1941) & $\mathrm{U}$ & $1(1-0)$ & $3(1-0-2)$ \\
\hline Salticus peckhamae (Cockerell 1897) & & $1(1-0)$ & $1(1-0-0)$ \\
\hline
\end{tabular}


TABLE 1. Continued.

\begin{tabular}{|c|c|c|c|}
\hline Family/species & $\begin{array}{c}\text { Status in } \\
\text { Washington }\end{array}$ & $\begin{array}{l}\text { Number of sites } \\
\text { collected from } \\
\text { (Pinus ponderosa- } \\
\text { P. monticola) }\end{array}$ & $\begin{array}{c}\text { Number of } \\
\text { specimens } \\
\text { (females-males- } \\
\text { juveniles) }\end{array}$ \\
\hline Sitticus dorsatus (Banks 1895) & $\mathrm{U}$ & $1(1-0)$ & $2(0-1-1)$ \\
\hline Unidentified juveniles & & $3(3-1)$ & $4(0-0-4)$ \\
\hline \multicolumn{4}{|l|}{ TetragnathidaE } \\
\hline Tetragnatha laboriosa Hentz 1850 & & $1(1-0)$ & $2(0-0-2)$ \\
\hline Tetragnatha sp. & & $1(1-0)$ & $1(0-0-1)$ \\
\hline \multicolumn{4}{|l|}{ THERIDIIDAE } \\
\hline Crustulina sticta (O. P.-Cambridge 1861) & & $2(2-0)$ & $2(0-0-2)$ \\
\hline Dipoena daltoni Levi 1953 & $\mathrm{R}$ & $1(1-0)$ & $1(0-1-0)$ \\
\hline Dipoena sp. \#1 & $\mathrm{R}$ & $1(1-0)$ & $1(1-0-0)$ \\
\hline Dipoena sp. & & $2(2-0)$ & $2(0-0-2)$ \\
\hline Enoplognatha intrepida (Sorensen 1898) & $\mathrm{U}$ & $1(1-0)$ & $1(1-0-0)$ \\
\hline Enoplognatha sp. & & $7(7-0)$ & $10(0-0-10)$ \\
\hline Euryopis formosa Banks 1908 & & $34(30-4)$ & $145(22-24-99)$ \\
\hline Steatoda sp. & & $8(7-1)$ & $42(0-0-42)$ \\
\hline Theridion rabuni Chamberlin and Ivie 1944 & $\mathrm{O}$ & $3(2-1)$ & $8(4-2-2)$ \\
\hline Theridion tinctum (Walckenaer 1802) & & $1(1-0)$ & $1(0-0-1)$ \\
\hline Theridion sp. & & $6(6-0)$ & $17(0-0-17)$ \\
\hline Unidentified juveniles & & $5(5-0)$ & $8(0-0-8)$ \\
\hline \multicolumn{4}{|l|}{ THOMISIDAE (SENSU LATO) } \\
\hline Apollophanes margareta Lowrie and Gertsch 1955 & & $2(1-1)$ & $2(0-0-2)$ \\
\hline Bassaniana utahensis (Gertsch 1932) & & $4(4-0)$ & $6(1-0-5)$ \\
\hline Ebo sp. & & $7(7-0)$ & $13(0-0-13)$ \\
\hline Misumenops sp. & & $2(2-0)$ & $2(0-0-2)$ \\
\hline Ozyptila yosemitica Schick 1965 & $\mathrm{R}$ & $1(1-0)$ & $1(1-0-0)$ \\
\hline Ozyptila sp. & & $2(1-1)$ & $3(0-0-3)$ \\
\hline Philodromus rufus Walckenaer 1826 & & $1(1-0)$ & $1(1-0-0)$ \\
\hline Philodromus spectabilis Keyserling 1880 & & $1(1-0)$ & $4(0-0-4)$ \\
\hline Philodromus sp. & & $3(2-1)$ & $35(0-0-35)$ \\
\hline Thanatus coloradensis Keyserling 1880 & $\mathrm{R}$ & $1(1-0)$ & $1(1-0-0)$ \\
\hline Thanatus formicinus (Clerck 1757) & $\mathrm{U}$ & $1(1-0)$ & $1(1-0-0)$ \\
\hline Thanatus sp. & & $2(2-0)$ & $2(0-0-2)$ \\
\hline Xysticus gulosus Keyserling 1880 & $\mathrm{U}$ & $1(1-0)$ & $1(1-0-0)$ \\
\hline Xysticus locuples Keyserling 1880 & & $5(5-0)$ & $8(5-2-1)$ \\
\hline Xysticus montanensis Keyserling 1887 & & $1(1-0)$ & $1(1-0-0)$ \\
\hline Xysticus sp. & & $7(7-0)$ & $9(0-0-9)$ \\
\hline \multicolumn{4}{|l|}{ TitanoEcidaE } \\
\hline Titanoeca nigrella (Chamberlin 1919) & & $4(4-0)$ & $6(0-0-6)$ \\
\hline \multicolumn{4}{|l|}{ ZORIDAE } \\
\hline Zora hespera Corey and Mott 1991 & $\mathrm{U}$ & $6(6-0)$ & $11(0-0-11)$ \\
\hline Juveniles not identified to family & & $5(5-0)$ & $5(0-0-5)$ \\
\hline
\end{tabular}

collected in eastern Washington, most in the vicinity of $P$. ponderosa or $P$. monticola. Additionally, E. formosa was absent from over 400 fallen P. ponderosa, P. monticola, and Pinus strobus L. cones sampled at 5 western Washington sites (data not shown).

Although we visually inspected most cones sampled prior to beating, most spiders were not visible until they had been expelled from the cone. Nevertheless, spiders were occasionally observed guarding an egg sac or occupying a prey capture web or retreat in the cones. Most inhabited retreats were made by agelenids, gnaphosids, and salticids. In addition, exuviae, unattended egg sacs, vacant prey capture webs, and retreats were commonly observed. Most of the identifiable exuviae were from Pardosa spp. (Lycosidae). Spiders placed egg sacs and retreats on the upper surface of cone scales and on the concave surface at the base of the cone. We often observed agelenid webs incorporating cones, with the sheet web covering the cone surface and the funnel receding between cone scales or beneath the cone.

Pinus ponderosa cones appeared to persist on the ground for at least 2 years before disintegrating. Recently fallen cones were often found among cones that had fallen during 
TABLE 2. Summary of spiders by family. Spiders were collected from fallen pine cones and ground-level non-cone microhabitats in eastern Washington. Cone data were collected from 70 sites, non-cone data from 39 sites where both cone and ground-level non-cone sampling was done.

\begin{tabular}{|c|c|c|c|c|}
\hline Family & Cone specimens & Cone sites & $\begin{array}{l}\% \text { of all cone } \\
\text { specimens }\end{array}$ & $\begin{array}{l}\% \text { of all ground-level } \\
\text { non-cone specimens }\end{array}$ \\
\hline Agelenidae (s.l.) & 43 & 19 & 4.1 & 2.8 \\
\hline Amaurobiidae & 31 & 11 & 2.9 & 3.0 \\
\hline Anyphaenidae & 37 & 13 & 3.5 & - \\
\hline Araneidae & 2 & 2 & 0.2 & 0.3 \\
\hline Clubionidae & 3 & 3 & 0.3 & 3.5 \\
\hline Corinnidae & 51 & 13 & 4.8 & 8.3 \\
\hline Cybaeidae & 3 & 2 & 0.3 & 1 \\
\hline Dictynidae & $105^{\mathrm{a}}$ & 19 & $9.91^{\mathrm{a}}$ & 5.1 \\
\hline Gnaphosidae & 143 & 38 & 13.5 & 3.8 \\
\hline Hahniidae & 3 & 1 & 0.3 & 0.2 \\
\hline Leptonetidae & - & - & - & 0.3 \\
\hline Linyphiidae & 143 & 40 & 13.5 & 44.0 \\
\hline Liocranidae & 3 & 3 & 0.3 & 2.0 \\
\hline Lycosidae & 35 & 18 & 3.3 & 9.1 \\
\hline Mimetidae & 1 & 1 & 0.1 & - \\
\hline Mysmenidae & 4 & 3 & 0.4 & 0.2 \\
\hline Oonopidae & 1 & 1 & 0.1 & - \\
\hline Oxyopidae & - & - & - & 0.2 \\
\hline Pholcidae & 50 & 15 & 4.7 & 1 \\
\hline Pimoidae & 7 & 5 & 0.7 & 0.2 \\
\hline Pisauridae & - & - & - & 0.3 \\
\hline Salticidae & 38 & 19 & 3.6 & 2.8 \\
\hline Telemidae & - & - & - & 1 \\
\hline Tetragnathidae & 3 & 2 & 0.3 & 0.5 \\
\hline Theridiidae & 242 & 47 & 22.8 & 3.8 \\
\hline Thomisidae (s.l.) & 90 & 31 & 8.5 & 2.8 \\
\hline Titanoecidae & 6 & 4 & 0.6 & 0.2 \\
\hline unknown & 5 & 5 & 0.5 & - \\
\hline Zoridae & 11 & 6 & 1.0 & 2.3 \\
\hline TOTAL & 1060 & - & 100 & 100 \\
\hline
\end{tabular}

aThis value was skewed by 63 Dictyna calcarata we collected from one site, a finding we consider an outlier.

each of the previous 2 years, judging from color changes and structural decay. We did not test whether cone age class correlated with the species or number of specimens collected from cones, but no obvious trends were observed in the field. Spiders were collected from newly fallen cones still sticky with resin, from cones that had fallen the previous year, and from older cones that disintegrated when beaten.

Fallen cones often provided the most extensive cover, and sometimes the only cover, for spiders at ground level. This was especially true in $P$. ponderosa stands, where the shrub and herbaceous layers were often sparse and litter was frequently thin to nonexistent. Litter, where it occurred, consisted primarily of pine needles, but occasionally also contained dried grass, bark scales, twigs, and, less commonly, deciduous leaves.

In addition to spiders, a number of other arthropods were found in fallen cones. These included harvestmen (Opiliones), pseudoscorpions (Pseudoscorpionida), mites (Acari), millipedes (Diplopoda), centipedes (Chilopoda), and a number of insect orders, such as Dermaptera (earwigs) and Heteroptera (true bugs) (Crawford unpublished data).

\section{Discussion}

Adding fallen cones to the spider microhabitats sampled in Washington State has increased the number of species found at most sampling sites, added new species to the state list, provided specimens of several rare spiders to the Burke Collection, and expanded our knowledge of habitat utilization by $E$. formosa and P. americana, as well as 87 other species (Table 1 ).

To our knowledge, the present study is the first to describe the spider fauna of fallen cones. The insect fauna of attached conifer seed cones (Turgeon et al. 1994) and fallen 


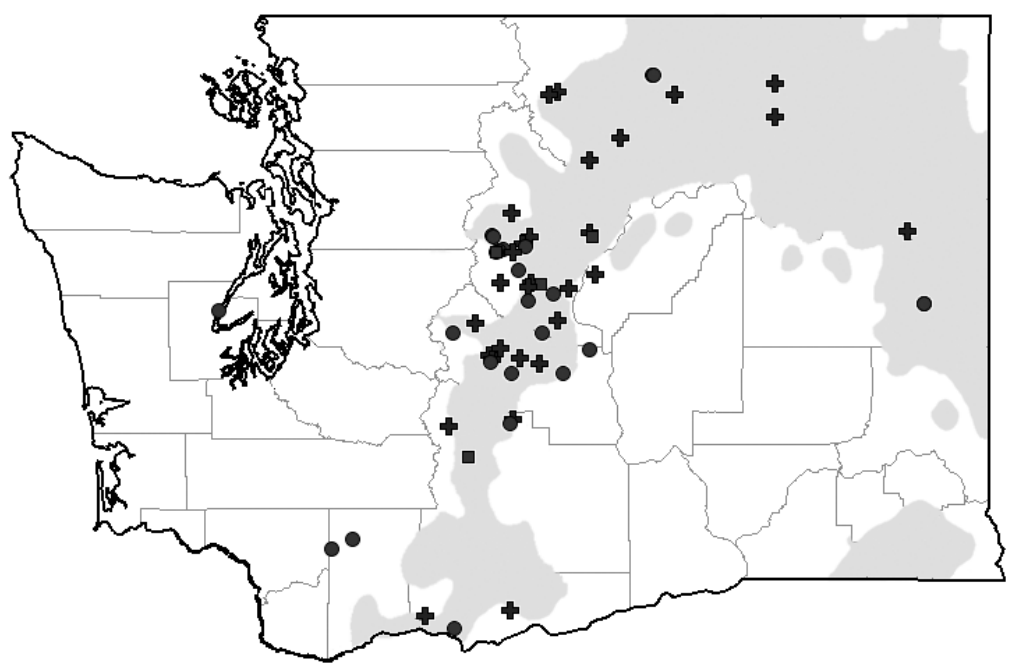

Fig. 2. Map of Washington showing sites where Euryopus formosa was collected from fallen pine cones (crosses), noncone microhabitats (circles), or both (squares). Shaded area indicates approximate distribution of Pinus ponderosa in eastern Washington (Kotok 1973).

pine cones (Wheeler and Stoops 2010) has been studied. However, there has been only passing mention of the presence of spiders in cones: Douglas-fir (Pseudotsuga menziesii [Mirb.] Franco) cones in Washington (Deyrup and Deyrup 1978) and cones of various pine species in Texas (Kulhavy and Baldridge 1987) and the southeastern United States (Wheeler and Stoops 2010).

Our finding that the mean number of spiders per cone varied widely from 0.02 to 1.3 between sampling sites indicates that spider occurrence in fallen cones is patchy. In support of this, we commonly observed in the field that a particular accumulation of cones apparently lacked any spiders, while another accumulation nearby would yield several individuals.

We assume that many of the species taken in cones only once were in the microhabitat by chance. This seems especially likely for the orb-weavers we collected, for example. Nevertheless, our findings indicate that cones are an important, perhaps even primary microhabitat for a few species, and are used temporarily by many others at different points in their life cycle. Where fallen cones were present, they added a significant structural component to the litter, which was often thin to nonexistent. Consequently, fallen cones provided a place for spiders to place egg sacs and retreats, molt, and capture prey, as evidenced by the spider traces found in and on cones. The cone habitat may also give spiders some protection from desiccation or predators. These hypotheses can be tested in the future.

Fallen cones were a predictable microhabitat in which to find Euryopis formosa and, to a lesser extent, Pholcophora americana, the 2 most numerous species collected from cones. Both co-occurred in cones at a significant number of sampling sites. These results, taken with the finding that just a few years of cone sampling was enough to significantly increase the number of specimens and collection site records for both species in the extensive Burke Collection, indicate that the species' presence in fallen cones was not random.

Prior to cone sampling, E. formosa had been collected from a variety of microhabitats in Washington, but never predictably (Burke Collection). Nevertheless, our discovery of its regular occurrence in fallen pine cones is consistent with those earlier records in that most collections from non-cone microhabitats were at sites in the $P$. ponderosa belt on the eastern slope of the state's Cascade Range. Gillette et al. (2008) identified E. formosa as a significant indicator of old-growth status in a pitfall study of the ground-dwelling spiders in P. ponderosa stands in the Cascade Range of California. Considering the wide distribution 
of P. ponderosa and P. monticola in Washington, E. formosa may be more common in the state than it previously appeared to be.

How $E$. formosa uses fallen cones remains unclear. Despite the species being common in our samples, we observed few in situ. Those few we did observe were never associated with silk structures. This observation is consistent with the report by Levi (1954) that spiders of the genus Euryopis do not spin webs. Although E. formosa and several other species of Euryopis are reputed ant predators (Porter and Eastmond 1982, Clark and Blom 1992), we did not observe ant predation by $E$. formosa. Furthermore, ant carcasses were rare in pine cone samples (L.J. Ramseyer personal observation).

Our discovery that Pholcophora americana occurs regularly in fallen cones in Washington is consistent with Gertsch (1982), who characterized the genus Pholcophora as living "close to the soil," in particular "under ground objects, in leaf and plant detritus, and in soil openings and caves." Specimens of $P$. americana in the Burke Collection were typically collected under rocks, boards, and bark as well. Conceivably, P. americana individuals inhabit fallen cones because of their close proximity to the soil and because cones provide spaces suitable for building prey capture webs. Gertsch (1982) reported that $P$. americana individuals "spin web tangles in dark spaces and remain there in close contact with such webs as permanent residents, often in informal colonies."

We collected 18 spider species that are rare in Washington from fallen cones (Table 1), mostly in such limited numbers, locations, and timeframes that the significance of their occurrence in the microhabitat remains an open question. However, we took a total of 6 mature and 2 juvenile specimens of Theridion rabuni from fallen cones at 3 different sites over a 3year period. This consistency, and the absence thus far of the species from other microhabitats in Washington, indicates that fallen pine cones may be a primary microhabitat for $T$. rabuni in the state.

Theridion rabuni is widespread outside of Washington, ranging from citrus groves in California (Carroll 1980) to soybean fields in Delaware (Culin 1978) and numerous states between (Levi and Randolph 1975). In contrast, all T. rabuni specimens taken from fallen cones in Washington were collected within an approximately $10-\mathrm{km}$ radius centered on the Chiwaukum Mountains in Chelan County (approx. $47.74^{\circ} \mathrm{N}, 120.91^{\circ} \mathrm{W}$ ). Further sampling is needed to determine whether T. rabuni is as localized in Washington as the present data suggest.

Pinus ponderosa is adapted to survive frequent low-intensity fires (Agee 1993). We suggest that cones dropped after a ground fire may provide crucial cover for surviving and recolonizing spiders in a way analogous to hay refugia placed in harvested agricultural fields (cf. Halaj et al. 2000). Our finding that fallen P. ponderosa cones persisted 2 or more years before disintegrating indicates that local spider populations probably have relatively consistent access to the fallen cone microhabitat in stands that have not burned recently. Presumably, the multiyear persistence of fallen cones would offset occasional years of poor cone production typical in $P$. ponderosa (Krannitz and Duralia 2004).

Our observations on E. formosa and P. americana, together with the marked differences in spider family abundances between the fallen cone and ground-level non-cone microhabitats (Table 2), indicate that the fallen cone microhabitat of the P. ponderosa zone of eastern Washington supports a distinct spider fauna. However, the Chao 1 estimator of species richness indicates that the 89 species we found in cones constitute only $62 \%$ of the estimated total number of species in the microhabitat at our study sites. Thus, further sampling is required to fully characterize the fallen cone spider assemblage of eastern Washington and its differences from nearby microhabitats.

Cone-dwelling spiders in Washington are not restricted to cones of the tree species reported here, nor is the fallen cone microhabitat unique to Washington State. In addition to the data presented here, we have also collected spiders from fallen cones of mountain hemlock (Tsuga mertensiana Bong. Carrier), Douglas-fir, lodgepole pine (Pinus contorta Dougl. var. latifolia Engelm.), and eastern white pine (P. strobus L.) in Washington (Ramseyer and Crawford unpublished data). Spiders have also been collected from fallen cones of $P$. ponderosa in California and P. strobus in Massachusetts (L.J. Ramseyer unpublished data) and reported as present in cones of various pine species in Texas (Kulhavy and Baldridge 1987) and the southeastern United States (Wheeler 
and Stoops 2010). Numerous other examples likely await discovery given that more than 70 species of conifers in North America drop open, intact cones (Thieret 1993, Earle 2013).

\section{ACKNOWLEDGMENTS}

The following Burke Museum volunteers (in chronological order) helped with curation of Washington pine cone spider specimens: Della Scott, Erin Wheeler, Andrea Maillet, Elizabeth Heckendorf, Erin Haley, Matisse Lorance, Lauren Taylor, and Cheryl Tsuhako. The authors did all other project work with no financial support.

\section{Literature Cited}

AgEe, J.K. 1993. Fire ecology of Pacific Northwest forests Island Press, Washington, DC. 499 pp.

Carroll, D.P. 1980. Biological notes on the spiders of some citrus groves in central and southern California. Entomological News 91:147-154.

Clark, W.H., AND P.E. Blom. 1992. Notes on spider (Theridiidae, Salticidae) predation of the harvester ant, Pogonomyrmex salinus Olsen (Hymenoptera: Formicidae: Myrmicinae), and a possible parasitoid fly (Chloropidae). Great Basin Naturalist 52:385-386.

Coddington, J.A., L.H. Young, And F.A. Coyle. 1996. Estimating spider species richness in a southern Appalachian cove hardwood forest. Journal of Arachnology 24:111-128.

Colwell, R.K. 2013. EstimateS: statistical estimation of species richness and shared species from samples. Version 9, User's guide and application. http://purl/ oclc.org/estimates

Crawford, R.L. 1988. An annotated checklist of the spiders of Washington. Burke Museum Contributions in Natural History and Anthropology 5:1-48.

Culin, J.D. 1978. Additional spiders (Araneae) of Delaware. Entomological News 89:145-146.

Daubenmire, R., and J. Daubenmire. 1968. Forest vegetation of eastern Washington and northern Idaho. Bulletin XT 0060, Washington State University, Agricultural Research Center. Reprinted 1984 by Cooperative Extension.

Deyrup, M., AND N. Deyrup. 1978. Pupation of Hemerobius in Douglas-fir cones. Pan-Pacific Entomologist 54:143-146.

DiCE, L.R. 1945. Measures of the amount of ecological association between species. Ecology 26:297-302.

EARLE, C.J. 2013. Gymnosperm database [online]. http:// conifers.org/
FrankLin, J.F., and C.T. Dyrness. 1973. Natural vegetation of Oregon and Washington. General Technical Report PNW-GTR-008, USDA Forest Service, Pacific Northwest Research Station, Portland, OR.

Gertsch, W.J. 1982. The spider genera Pholcophora and Anopsicus (Araneae, Pholcidae) in North America, Central America and the West Indies. Association for Mexican Cave Studies Bulletin 8:95-144.

Gillette, N.E., R.S. Vetter, S.R. Mori, C.R. Rudolph, AND D.R. WELTY. 2008. Response of ground-dwelling spider assemblages to prescribed fire following stand structure manipulation in the southern Cascade Range. Canadian Journal of Forest Research 38:969-980.

Halaj, J., A.B. Cady, and G.W. UETZ. 2000. Modular habitat refugia enhance generalist predators and lower plant damage in soybeans. Environmental Entomology 29:383-393.

KotoK, E.S. 1973. Ponderosa pine . . an American wood. USDA Forest Service [Bulletin] FS-254. 6 pp.

Krannitz, P.G., and T.E. Duralia. 2004. Cone and seed production in Pinus ponderosa: a review. Western North American Naturalist 64:208-218.

KulhaVy, D.L., AND R.S. BaLdRIDGE. 1987. Life in a pine cone. Ward's Bulletin (Fall). 2 pp.

Levi, H.W. 1954. Spiders of the genus Euryopis from North and Central America (Araneae, Theridiidae). American Museum Novitates 1666:1-48.

LEVI, H.W., AND D.E. RandolPH. 1975. A key and checklist of American spiders of the family Theridiidae north of Mexico (Araneae). Journal of Arachnology 3:31-51.

Porter, S.D., AND D.A. Eastmond. 1982. Euryopis coki (Theridiidae), a spider that preys on Pogonomyrmex ants. Journal of Arachnology 10:275-277.

Thieret, J.W. 1993. Pinaceae. In: Flora of North America Editorial Committee, editors, Flora of North America north of Mexico. Volume 2. New York and Oxford. http://www.efloras.org/florataxon.aspx?flora_id $=1 \& t$ axon id $=10691$

Turgeon, J.J., A. Roques, And P. DE Groot. 1994. Insect fauna of coniferous seed cones: diversity, host plant interactions, and management. Annual Review of Entomology 39:179-212.

Wheeler, A.G., JR., AND C.A. Stoops. 2010. Cnemodus hirtipes Blatchley and C. mavortius (Say) (Hemiptera: Lygaeoidea: Rhyparochromidae) in fallen pine cones, with consideration of the biological significance of cone occupancy. Proceedings of the Entomological Society of Washington 112:155-168.

World Spider Catalog. 2014. World Spider Catalog, version 15.5 [online]. Natural History Museum of Bern, Bern, Switzerland; [accessed 29 August 2014]. http://wsc.nmbe.ch

Received 14 February 2014 Accepted 17 October 2014 